\title{
SAÚDE MENTAL NA ATENÇÃO PRIMÁRIA À SAUDE
}

\author{
MENTAL HEALTH IN PRIMARY HEALTH CARE
}

\author{
Mauri Gonçalves Junior ${ }^{\mathrm{a}^{*}}$, Gabriela Camargo Tobias ${ }^{\mathrm{b}^{*}}$, Cristiane Chagas Teixeira ${ }^{\mathrm{c}^{*}}$ \\ maurijunior12@hotmail.comª ,gabicamargo22@gmail.com ${ }^{\mathrm{b}}$,cc-teixeira@hotmail.com \\ Universidade Federal de Goiás*
}

\section{RESUMO}

Objetivo: identificar evidências da literatura referente a gestão do cuidado com o paciente em sofrimento mental na Atenção Primária à Saúde e as intervenções eficazes para o paciente em sofrimento psíquico. Método: revisão integrativa da literatura, entre 2007 a 2017, nas bases de dados Scielo, Cochrane, Lilacs e Medline/ PubMed. Os descritores utilizados foram "Atenção Primária à Saúde”, “Saúde Mental”, "Cuidado". Resultados: identificaram-se 18 artigos no período estudado. Os estudos apresentaram conceitos como "acolhimento" e "matriciamento", ambos com fundamentos para o cuidado integral e resolutivo de paciente em sofrimento mental. Conclusão: constatou-se que o cuidado ao paciente em sofrimento mental não necessita de inúmeros recursos tecnológicos/sofisticadas ou mudanças nos processos de trabalhos, pois, o foco do cuidado está na postura do profissional e da organização da rede de atenção psicossocial como espaço de transição para o paciente receber o cuidado que necessita.

Palavras-chave: Atenção primária à saúde; saúde mental; assistência centrada no paciente

\section{ABSTRACT}

Objective: to identify evidence from the literature regarding the management of patient care in mental suffering in Primary Health Care and effective interventions for the patient in psychological distress. Method: integrative review of the literature, from 2007 to 2017, in the databases Scielo, Cochrane, Lilacs and Medline / PubMed. The descriptors used were "Primary Health Care", "Mental Health", "Care". Results: 18 articles were identified in the study period. The studies presented concepts such as "reception" and "matriciamento", both with bases for the integral and resolutive care of patient in mental suffering. Conclusion: it was observed that care for the mentally ill patient does not require innumerable technological / sophisticated resources or changes in work processes, since the focus of care lies in the professional's posture and the organization of the psychosocial care network as a space for transition for the patient to receive the care they need.

Keywords: Primary health care; mental health; patient-centered care 


\section{Introdução}

A Atenção Primária à Saúde (APS) possibilita acesso das pessoas ao Sistema Único de Saúde (SUS), inclusive daquelas que demandam cuidado em saúde mental. As ações são desenvolvidas em território geograficamente conhecido, possibilitando aos profissionais de saúde proximidade para conhecer a história de vida das pessoas e de seus vínculos com a comunidade/ território onde moram, bem como, outros elementos dos contextos de vida ${ }^{1}$.

Neste contexto, a saúde mental adquiri fortalecimento através de ações de promoção e prevenção, devido ao aumento das taxas de suicídio, pacientes acometidos por transtorno psicopatológico e novas drogas terapêuticas. O crescimento de pacientes em sofrimento mental chama atenção dos governos e instituições para reflexão sobre o cuidado em saúde mental ${ }^{2}$.

O sofrimento mental tem se tornado alvo de inúmeras pesquisas e debates em nossa sociedade, pois cresce cada vez mais o número de pessoas que apresentam esta demanda nos consultórios, ambulatórios e Unidades Básicas de Saúde. A etiologia do sofrimento mental perpassa por vários fatores, sejam eles biológicos, sociais, psicopatológicos, uso abusivo de drogas lícitas e ilícitas, questões financeiras/econômicas ou todos eles ao mesmo tempo ${ }^{3}$.

As equipes das Estratégias de Saúde da Família (ESF) devem estar preparadas para receber toda e qualquer demanda das famílias sob responsabilidade de saúde mental ou não. Levando em consideração os princípios do SUS, a saúde tem que ser garantida mediante oferta de condições de acessibilidade e assistência equânime, integral e universal ${ }^{4}$.

As intervenções em saúde mental promovem novas possibilidades de modificar e qualificar as condições e modos de vida, orientando-se pela produção de vida e saúde, não se restringindo à cura de doenças. Isso significa acreditar que a vida pode ter várias formas de ser percebida, experimentada e vivida. Para tanto, é necessário olhar o sujeito em suas múltiplas dimensões, com seus desejos, anseios, valores e escolhas ${ }^{1,5}$.
$\mathrm{Na}$ APS, o desenvolvimento de intervenções em saúde mental é construído no cotidiano dos encontros entre profissionais e usuários, em que ambos criam novas ferramentas e estratégias para compartilhar e construir juntos o cuidado em saúde ${ }^{1}$.

A falta de acolhimento da pessoa em sofrimento mental pelos profissionais de saúde que atuam no serviço público, sobretudo, por aqueles que atuam na atenção básica, constituise uma dificuldade que pode ser identificada em vários municípios de pequeno e grande porte, e, normalmente, está relacionada à falta de capacitação dos profissionais para cuidar desta clientela, até mesmo quando a queixa refere-se a problemas físicos para os quais existem protocolos de atendimento definido, como é o caso da hipertensão e diabetes ${ }^{4}$.

O desafio que se coloca é a criação de circuitos paralelos e protegidos de vida para usuários e sociedade. Isso leva o tema da saúde mental para além do SUS, já que para realizar-se implica na abertura da sociedade para sua própria diversidade ${ }^{6}$.

Contudo, diante do aumento significativo de pacientes em sofrimento mental que buscam na APS resolução para o sofrimento, este estudo tem como hipótese o desconhecimento de grande parte dos profissionais da APS sobre as diretrizes para manejo dos pacientes em sofrimento mental, produzindo arranjos disfuncionais pouco resolutivos e que reforçam cada vez mais o modelo hospitalocêntrico no cuidado destes usuários.

Portanto, torna-se relevante aos profissionais de saúde conhecerem esta realidade e reconhecerem sua responsabilidade ao enfrentamento desta problemática. Como grande parte da população brasileira utiliza o serviço público de saúde, a APS se torna porta de entrada para estes usuários, diante dessa premissa, como os profissionais irão receber esta demanda? Como se dará o cuidado? É de extrema importância conhecer esta problemática para que desenvolvam ações terapêuticas mais resolutivas e integrativas.

Procurando contribuir e somar esforços para melhoria da assistência em saúde mental, propôs-se a presente investigação com objetivo de identificar evidências da literatura referente a gestão do cuidado com o paciente em 
sofrimento mental na Atenção Primária à Saúde $\mathrm{e}$ as intervenções eficazes para o paciente em sofrimento psíquico.

\section{Metodologia}

Trata-se de uma revisão integrativa da literatura, que consiste em uma ampla abordagem metodológica referente às revisões, permitindo inclusão de estudos experimentais e nãoexperimentais para uma compreensão completa do fenômeno analisado. Inclui também dados da literatura teórica e empírica, além de incorporar um vasto leque de propósitos, definição de conceitos, revisão de teorias, evidências e análise de problemas metodológicos de um tópico particular $^{7}$

Os estudos de revisão da literatura devem apresentar uma análise crítica, ponto de vista ou avaliação que favoreça a discussão de novas ideias ou perspectivas, sobre temas de relevância para o conhecimento pedagógico, científico, universitário ou profissional ${ }^{7}$.

Para a elaboração da revisão integrativa as seguintes etapas foram percorridas: estabelecimento da hipótese e objetivos da revisão integrativa; estabelecimento de critérios de inclusão e exclusão de artigos (seleção da amostra); definição das informações a serem extraídas dos artigos selecionados; análise dos resultados; discussão e apresentação dos resultados ${ }^{7-8}$.

A questão de pesquisa delimitada foi: o que se tem publicado sobre a gestão do cuidado com pacientes em sofrimento mental na Atenção Primária à Saúde? Quais são as intervenções eficazes no cuidado com paciente em sofrimento psíquico?

Para localização e seleção dos estudos foi realizada nas bases de dados: Scientific Electronic Library Online (Scielo), Cochrane, Literatura Latino-Americana e do Caribe em Ciências da Saúde (LILACS) e Medical Literature Analysis and Retrieval System Online (MEDLINE/ PubMed).

Os critérios de inclusão foram: artigos publicados em português, com os resumos disponíveis nas bases de dados selecionadas, no período compreendido entre 2007 a 2017; artigos que retratassem procedimentos, intervenções ou diretrizes sobre a gestão do cuidado com paciente em sofrimento mental na Atenção Primária à Saúde. Foram excluídas dissertações e teses, e artigos que apresentavam duplicidade, ou seja, publicações recuperadas em mais de uma das bases de dados.

Os descritores utilizados foram "Atenção Primária de Saúde", "Saúde Mental", "Cuidado" de forma isolada e combinada. A busca foi realizada utilizando os critérios de inclusão, a amostra final foi constituída de 18 artigos, conforme Figura 1.

Figura 1 - Fluxograma do processo de seleção dos estudos.

\begin{tabular}{|c|c|c|c|}
\hline \multirow[t]{2}{*}{$\begin{array}{l}\text { Scielo } \\
(n=61)\end{array}$} & $\begin{array}{c}\text { Cochrane } \\
(\mathrm{n}=41)\end{array}$ & $\begin{array}{c}\text { Lilacs } \\
(\mathrm{n}=206)\end{array}$ & $\begin{array}{c}\text { Medline/Pubmed } \\
\qquad(\mathrm{n}=116)\end{array}$ \\
\hline & \multicolumn{3}{|c|}{ Artigos potencialmente relevantes $(n=424)$} \\
\hline & $\begin{array}{l}\text { Artigos Excluídos } \\
\text { Critérios de Exclu } \\
\text { a) Dissertações } \\
\text { b) Artigos que a } \\
\text { c) Artigos em lí } \\
\text { d) Artigos publi } \\
\text { e) Artigos publi }\end{array}$ & $\begin{array}{l}\text { duplicida } \\
\text { eira } \\
\text { os anteric } \\
\text { os poster }\end{array}$ & $\begin{array}{l}07 \\
17\end{array}$ \\
\hline \multicolumn{4}{|c|}{ Artigos Selecionados para revisão $(n=18)$} \\
\hline
\end{tabular}


Para análise e posterior síntese dos artigos que atenderam aos critérios de inclusão foi utilizado quadro construído para este fim, que contemplou os seguintes aspectos: base de dados, título, autores, resultados e ano de publicação.

A apresentação dos resultados e discussão dos dados obtidos foi realizada de forma descritiva, após leitura crítica dos artigos encontrados possibilitando ao leitor avaliação da aplicabilidade da revisão integrativa, de forma a atingir o objetivo proposto, ou seja, impactar positivamente na qualidade da prática de saúde, fornecendo subsídios ao profissional na tomada de decisão cotidiana e possibilitando a construção de novos olhares e sentidos para o fenômeno investigado.

\section{Resultados}

Analisou-se 18 estudos que atenderam aos critérios de inclusão previamente estabelecidos. Quanto às bases de dados incluídas na pesquisa, 16 foram localizados na base Scielo, um (1) na Lilacs e um (1) na Medline/Pubmed. Nas demais bases não foram localizados estudos que se enquadrassem aos critérios de inclusão.

Dentre os estudos incluídos apresentouse variação considerável sobre formação dos pesquisadores e sua participação nos estudos encontrados. Houve predominância de pesquisadores com formação em Psicologia, com 67\%, em seguida Enfermagem (16\%), Medicina (9\%), Terapia Ocupacional (6\%) e Educação Física (2\%). Em relação ao tipo de revista científica nas quais foram publicados os estudos incluídos na revisão, 65\% estudos foram publicados em revistas de Saúde Coletiva, 22\% estudos em revistas de Psicologia, $11 \%$ estudos em revista de Enfermagem, 6\% estudos em revistas de Saúde Mental e 5\% estudos em revista sobre Ciências Humanas.

Quanto ao tipo de delineamento de pesquisa dos estudos avaliados, evidenciouse na amostra $67 \%$ de estudos de campo, $22 \%$ revisões narrativas, $6 \%$ estudos de caso e $5 \%$ revisão sistemática da literatura. Em relação ao período de publicação, os anos de 2009 e 2017 apresentaram 22,2\%, os anos de 2011;2012; 2015 e 2016 apresentaram 11\% e os anos de 2008 e 2013 apresentaram 5,6\%.

$\mathrm{O}$ quadro abaixo segue informativo com base de dados, título, autores, resultados e ano de publicação, para melhor visibilidade dos dados obtidos, visando à reflexão crítica sobre a temática investigada.

Quadro 1 - Apresentação da síntese de estudos incluídos na revisão integrativa.

\begin{tabular}{|c|c|c|c|c|}
\hline $\begin{array}{c}\text { BASE DE } \\
\text { DADOS }\end{array}$ & TÍTULO & AUTORES & RESULTADOS & $\begin{array}{c}\text { ANO DE } \\
\text { PUBLICAÇÃO }\end{array}$ \\
\hline SCIELO & $\begin{array}{l}\text { Os CAPS e o trabalho } \\
\text { em rede: tecendo o apoio } \\
\text { matricial na atenção básica. }\end{array}$ & $\begin{array}{c}\text { Bezerra E, } \\
\text { Dimenstein M }\end{array}$ & $\begin{array}{l}\text { Articulação precária entre } \\
\text { os CAPS e a rede de } \\
\text { atenção básica, necessidade } \\
\text { de repensar a função dos } \\
\text { CAPS na rede e rever o } \\
\text { modelo de funcionamento } \\
\text { ambulatorial pregnante } \\
\text { e a formação acadêmica } \\
\text { dos profissionais, que se } \\
\text { revela insatisfatória para o } \\
\text { cuidado aos portadores de } \\
\text { transtornos mentais. }\end{array}$ & 2008 \\
\hline
\end{tabular}




\begin{tabular}{|c|c|c|c|c|}
\hline SCIELO & $\begin{array}{l}\text { Desinstitucionalização dos } \\
\text { cuidados a pessoas com } \\
\text { transtornos mentais na } \\
\text { atenção básica: aportes para } \\
\text { a implementação de ações. }\end{array}$ & $\begin{array}{l}\text { Vecchia MD, } \\
\text { Martins STF }\end{array}$ & $\begin{array}{c}\text { Desenvolver processos de } \\
\text { comunicação que visem } \\
\text { ampliar a legibilidade } \\
\text { profissional; Superar a } \\
\text { centralização em ações } \\
\text { restritas aos enquadres } \\
\text { tradicionais; Manter } \\
\text { questionamento permanente } \\
\text { com relação ao risco de } \\
\text { psiquiatrização do cuidado } \\
\text { em saúde mental; Superar } \\
\text { concepções culpabilizantes } \\
\text { do grupo familiar e investir } \\
\text { na formação das equipes } \\
\text { de atenção básica para as } \\
\text { múltiplas dimensões do } \\
\text { cuidado em saúde mental. }\end{array}$ & 2009 \\
\hline SCIELO & $\begin{array}{c}\text { Parceria entre CAPS e PSF: } \\
\text { o desafio da construção de } \\
\text { um novo saber. }\end{array}$ & $\begin{array}{c}\text { Delfini PSS, } \\
\text { et al }\end{array}$ & $\begin{array}{c}\text { Grande demanda por } \\
\text { serviços de saúde e falta de } \\
\text { diretrizes institucionais para } \\
\text { a efetivação da parceria. } \\
\text { Em relação às pessoas } \\
\text { atendidas, o olhar voltado à } \\
\text { família e ao contexto social } \\
\text { aponta resultados positivos } \\
\text { em relação aqueles cujo } \\
\text { olhar é voltado somente } \\
\text { à doença. O trabalho } \\
\text { conjunto enriquece ainda } \\
\text { mais a prática e possibilita } \\
\text { uma rede maior de cuidados } \\
\text { no território. }\end{array}$ & 2009 \\
\hline SCIELO & $\begin{array}{l}\text { Concepções dos cuidados } \\
\text { em saúde mental por uma } \\
\text { equipe de saúde da família, } \\
\text { em perspectiva histórico- } \\
\text { cultural. }\end{array}$ & $\begin{array}{l}\text { Vecchia MD, } \\
\text { Martins STF }\end{array}$ & $\begin{array}{c}\text { A equipe considera } \\
\text { relevante a determinação } \\
\text { das condições de vida no } \\
\text { processo saúde-doença } \\
\text { da população atendida, a } \\
\text { necessidade de lançar mão } \\
\text { de estratégias diversificadas } \\
\text { no cuidado para além da } \\
\text { consulta, a importância } \\
\text { de se cuidar da saúde } \\
\text { mental da própria equipe, } \\
\text { bem como dificuldades na } \\
\text { abordagem da família. }\end{array}$ & 2009 \\
\hline
\end{tabular}




\begin{tabular}{|c|c|c|c|c|}
\hline LILACS & $\begin{array}{c}\text { Ações em saúde mental } \\
\text { na atenção primária no } \\
\text { município de Florianópolis, } \\
\text { Santa Catarina. }\end{array}$ & $\begin{array}{c}\text { Baroni DPM, } \\
\text { Fontana LM }\end{array}$ & $\begin{array}{l}\text { Surgimento de estudos em } \\
\text { saúde mental, embora os } \\
\text { relatos pareçam indicar } \\
\text { que as equipes ainda } \\
\text { desenvolvam suas ações } \\
\text { direcionadas mais para a } \\
\text { realização diagnóstica e } \\
\text { reabilitação do que para } \\
\text { a promoção em saúde } \\
\text { e prevenção de agravos } \\
\text { maiores decorridos do } \\
\text { sofrimento psíquico. }\end{array}$ & 2009 \\
\hline SCIELO & $\begin{array}{c}\text { Um estudo sobre a } \\
\text { itinerância como estratégia } \\
\text { de cuidado no contexto das } \\
\text { políticas públicas de saúde } \\
\text { no Brasil. }\end{array}$ & $\begin{array}{l}\text { Lemke RA, } \\
\text { Silva RAN }\end{array}$ & $\begin{array}{l}\text { No campo delicado de } \\
\text { articulação entre essas } \\
\text { políticas, as práticas } \\
\text { itinerantes passaram a ter } \\
\text { uma importância estratégica } \\
\text { na desinstitucionalização } \\
\text { das práticas e na construção } \\
\text { da integralidade do } \\
\text { cuidado. Tomou-se a } \\
\text { desinstitucionalização e } \\
\text { a integralidade como os } \\
\text { operadores conceituais que } \\
\text { marcam a diferença que } \\
\text { as reformas Psiquiátrica } \\
\text { e Sanitária pretendem } \\
\text { imprimir nas práticas de } \\
\text { cuidado. Ao ingressar } \\
\text { numa postura de busca } \\
\text { ativa no território de vida } \\
\text { dos usuários, as práticas } \\
\text { itinerantes se inserem } \\
\text { num campo de tensões, } \\
\text { no qual podem tanto ser } \\
\text { convocadas a funcionar } \\
\text { como uma peça do aparelho } \\
\text { de Estado no controle da } \\
\text { população, como se situar } \\
\text { num lugar estratégico para } \\
\text { a construção de um cuidado } \\
\text { contextualizado aos modos } \\
\text { de vida dos usuários. }\end{array}$ & 2011 \\
\hline
\end{tabular}




\begin{tabular}{|c|c|c|c|c|}
\hline SCIELO & $\begin{array}{l}\text { "Grupo" de artesanato: } \\
\text { espaço favorável à promoção } \\
\text { da saúde mental. }\end{array}$ & $\begin{array}{c}\text { Scardoelli } \\
\text { MGC, } \\
\text { Waidman MAP }\end{array}$ & $\begin{array}{l}\text { Foram encontrados três } \\
\text { categorias: Propiciando a } \\
\text { promoção da saúde mental; } \\
\text { Encontrando um espaço que } \\
\text { promove a fala, a escuta } \\
\text { e a partilha e; Buscando } \\
\text { um espaço de ensino/ } \\
\text { aprendizado. Participar } \\
\text { do grupo contribuiu para } \\
\text { melhoria da qualidade } \\
\text { de vida destas mulheres } \\
\text { que se encontravam em } \\
\text { situação de sofrimento } \\
\text { emocional, oferecendo } \\
\text { condições favoráveis ao } \\
\text { desenvolvimento do ponto } \\
\text { de vista psicossocial. }\end{array}$ & 2011 \\
\hline SCIELO & $\begin{array}{c}\text { A interlocução da saúde } \\
\text { mental com atenção básica } \\
\text { no município de Vitoria/ES. }\end{array}$ & $\begin{array}{c}\text { Rodrigues ES, } \\
\text { Moreira MIB }\end{array}$ & $\begin{array}{l}\text { A referida interlocução } \\
\text { ocorre de forma diferente } \\
\text { no território, com } \\
\text { aproximações importantes, } \\
\text { mas com grandes desafios. } \\
\text { Entretanto, as ricas } \\
\text { possibilidades já podem ser } \\
\text { percebidas com indicações } \\
\text { de que é preciso investir e } \\
\text { acreditar na potencialidade } \\
\text { da constituição de uma } \\
\text { rede substitutiva entre os } \\
\text { CAPS e a atenção básica, } \\
\text { como forma de garantir a } \\
\text { integralidade aos usuários } \\
\text { com transtorno mental. }\end{array}$ & 2012 \\
\hline SCIELO & $\begin{array}{l}\text { Saúde mental na estratégia } \\
\text { saúde da família: revisão da } \\
\text { literatura brasileira. }\end{array}$ & $\begin{array}{l}\text { Souza LGS, } \\
\text { et al }\end{array}$ & $\begin{array}{l}\text { Problemas como visões } \\
\text { estereotipadas sobre } \\
\text { os transtornos mentais, } \\
\text { predominância da } \\
\text { lógica manicomial, } \\
\text { ausência de registros, } \\
\text { fluxos, estratégias, apoio } \\
\text { qualificado às famílias e de } \\
\text { integração em rede. }\end{array}$ & 2012 \\
\hline
\end{tabular}




\begin{tabular}{|c|c|c|c|c|}
\hline SCIELO & $\begin{array}{c}\text { Itinerários de construção } \\
\text { de uma lógica territorial do } \\
\text { cuidado. }\end{array}$ & $\begin{array}{l}\text { Lemke RA, } \\
\text { Silva RAN }\end{array}$ & $\begin{array}{l}\text { As práticas de cuidado } \\
\text { dos acompanhantes } \\
\text { terapêuticos, agentes } \\
\text { comunitários de saúde } \\
\text { e redutores de danos na } \\
\text { construção de uma lógica } \\
\text { territorial do cuidado, } \\
\text { vinculam-se de modo } \\
\text { estreito aos desafios } \\
\text { lançados pelo movimento } \\
\text { de Reforma Psiquiátrica } \\
\text { e de Reforma Sanitária, } \\
\text { presentes nas atuais } \\
\text { políticas de Saúde Mental e } \\
\text { Atenção Básica. Ao operar } \\
\text { a partir dos conceitos de } \\
\text { desinstitucionalização e de } \\
\text { integralidade, essas práticas } \\
\text { guardam um importante } \\
\text { grau de transversalidade } \\
\text { no contexto das referidas } \\
\text { políticas e estão em } \\
\text { posição estratégica para a } \\
\text { construção de um modelo } \\
\text { de cuidado que tenha o } \\
\text { território como seu eixo } \\
\text { articulador. }\end{array}$ & 2013 \\
\hline SCIELO & $\begin{array}{c}\text { Saúde Mental na Atenção } \\
\text { Básica: Sentidos Atribuídos } \\
\text { pelos Agentes Comunitários } \\
\text { de Saúde. }\end{array}$ & $\begin{array}{c}\text { Moura RFS, } \\
\text { Silva CR }\end{array}$ & $\begin{array}{l}\text { A centralização dos } \\
\text { cuidados em Saúde } \\
\text { Mental nos CAPS, } \\
\text { especialmente através de } \\
\text { medicações. Em relação ao } \\
\text { Programa de Residência } \\
\text { Multiprofissional em } \\
\text { Saúde da UNIFESP-BS } \\
\text { (PRMAS), percebe-se o } \\
\text { papel de matriciamento } \\
\text { exercido pela residência, } \\
\text { por meio da escuta e } \\
\text { acompanhamento do } \\
\text { Agente Comunitário de } \\
\text { Saúde (ACS), que encontra } \\
\text { situações de sofrimento } \\
\text { psíquico em seu cotidiano } \\
\text { mas se sente despreparado } \\
\text { para lidar com elas. }\end{array}$ & 2015 \\
\hline
\end{tabular}




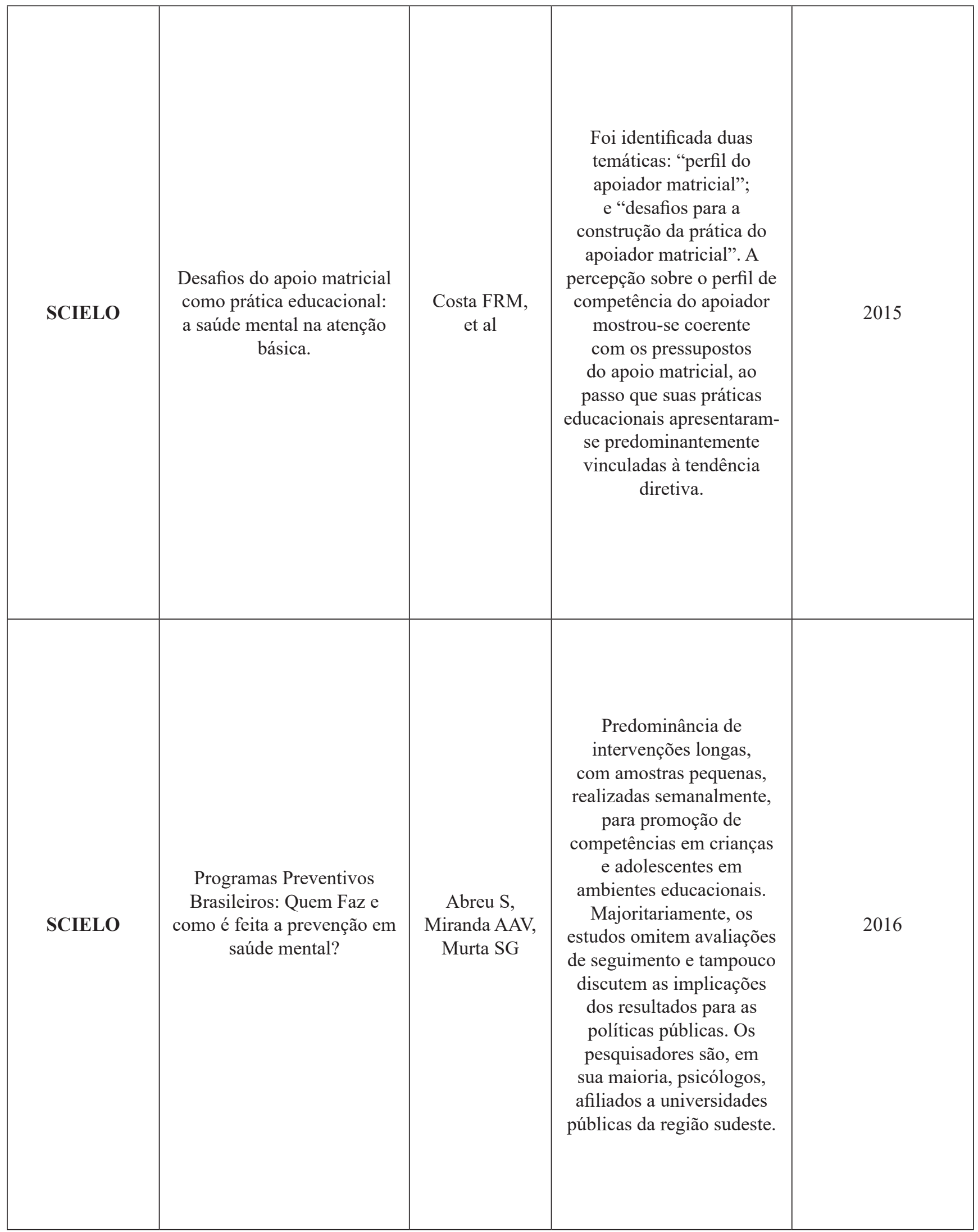




\begin{tabular}{|c|c|c|c|c|}
\hline $\begin{array}{l}\text { MEDLINE/ } \\
\text { PUBMED }\end{array}$ & $\begin{array}{l}\text { Sofrimento psíquico e a } \\
\text { abordagem da comunidade } \\
\text { na voz do agente } \\
\text { comunitário de saúde. }\end{array}$ & Paiva PC, et al & $\begin{array}{l}\text { Descreveu-se como a } \\
\text { comunidade percebe a } \\
\text { pessoa em sofrimento } \\
\text { psíquico e como está } \\
\text { se posiciona frente às } \\
\text { problemáticas do seu } \\
\text { cotidiano, no que se refere } \\
\text { a rejeição, preconceito, } \\
\text { discriminação e perda de } \\
\text { identidade. Destaca-se } \\
\text { que, pelo fato de estar } \\
\text { inserido na comunidade, } \\
\text { o agente comunitário de } \\
\text { saúde percebe de forma } \\
\text { mais apurada como tal } \\
\text { grupo social trata a pessoa } \\
\text { em sofrimento mental. } \\
\text { A rejeição da pessoa que } \\
\text { adoeceu é observada } \\
\text { como uma reação bastante } \\
\text { comum, acompanhada } \\
\text { do preconceito } \\
\text { e discriminação, } \\
\text { marginalizando-a da } \\
\text { sociedade. }\end{array}$ & 2016 \\
\hline SCIELO & $\begin{array}{c}\text { Saúde mental e atenção } \\
\text { básica: território, violência } \\
\text { e o desafio das abordagens } \\
\text { psicossociais. }\end{array}$ & $\begin{array}{c}\text { Prata NISS } \\
\text { et al }\end{array}$ & $\begin{array}{l}\text { Expansão acelerada } \\
\text { da Estratégia Saúde da } \\
\text { Família com impactos } \\
\text { no processo de trabalho, } \\
\text { discurso dos gestores com } \\
\text { evidência de abertura para } \\
\text { inclusão da saúde mental na } \\
\text { Estratégia, narrativas dos } \\
\text { trabalhadores explicitando } \\
\text { sensação de despreparo } \\
\text { e baixa percepção do } \\
\text { potencial terapêutico da } \\
\text { atenção básica, violência } \\
\text { nos territórios causando } \\
\text { tensões e ambivalências em } \\
\text { relação aos poderes locais, } \\
\text { associação direta entre } \\
\text { saúde mental e cotidiano } \\
\text { violento. }\end{array}$ & 2017 \\
\hline
\end{tabular}




\begin{tabular}{|c|c|c|c|c|}
\hline SCIELO & $\begin{array}{l}\text { Ações de saúde mental na } \\
\text { rede de atenção psicossocial } \\
\text { pela perspectiva dos } \\
\text { usuários. }\end{array}$ & $\begin{array}{c}\text { Moreira MIB, } \\
\text { Onocko- } \\
\text { Campos RT }\end{array}$ & $\begin{array}{l}\text { Desafios na implementação } \\
\text { de ações de saúde mental } \\
\text { na atenção básica e em } \\
\text { diferentes pontos da rede } \\
\text { psicossocial, visto que } \\
\text { os usuários reconhecem } \\
\text { a existência de uma rede } \\
\text { de saúde, mas indicam } \\
\text { acesso limitado a ela, pois } \\
\text { entendem que as ações } \\
\text { poderiam ser realizadas em } \\
\text { espaços facilitadores de } \\
\text { sua aproximação e inserção } \\
\text { na comunidade. Há } \\
\text { fragilidade na articulação e } \\
\text { na efetivação de cuidados } \\
\text { integrados nos serviços } \\
\text { e, consequentemente, as } \\
\text { demandas de saúde mental } \\
\text { se mantêm centralizadas } \\
\text { nos serviços especializados, } \\
\text { demonstrando forte inibição } \\
\text { do trabalho em rede. }\end{array}$ & 2017 \\
\hline SCIELO & $\begin{array}{l}\text { Em busca do comum: } \\
\text { o cuidado do agente } \\
\text { comunitário de saúde em } \\
\text { Saúde Mental. }\end{array}$ & $\begin{array}{l}\text { Saffer DA, } \\
\text { Barone LR }\end{array}$ & $\begin{array}{l}\text { Os Agentes Comunitários } \\
\text { de Saúde enredados na } \\
\text { trama do território afetam- } \\
\text { se pelo que é seu e podem } \\
\text { agir a partir desses afetos. } \\
\text { Entretanto, os modos de } \\
\text { composição entre vida } \\
\text { pessoal, saber comunitário } \\
\text { e prática profissional } \\
\text { podem ter vários } \\
\text { resultados, gerando também } \\
\text { ações culpabilizantes } \\
\text { e higienistas, sendo } \\
\text { necessária atenção } \\
\text { constante às direções éticas } \\
\text { pelas quais se constrói o } \\
\text { trabalho. }\end{array}$ & 2017 \\
\hline
\end{tabular}




\begin{tabular}{|c|c|c|c|c|}
\hline SCIELO & $\begin{array}{c}\text { O atendimento psicológico } \\
\text { ao paciente com diagnóstico } \\
\text { de depressão na Atenção } \\
\text { Básica. }\end{array}$ & $\begin{array}{l}\text { Motta CCL, } \\
\text { More CLOO, } \\
\text { Nunes CHSS }\end{array}$ & $\begin{array}{c}\text { O atendimento na rede } \\
\text { de Atenção Básica } \\
\text { delimita o tratamento de } \\
\text { quadros depressivos sob } \\
\text { a perspectiva sobretudo } \\
\text { biológica; a eleição da } \\
\text { psicoterapia individual } \\
\text { como principal forma } \\
\text { de atendimento remete } \\
\text { a práticas tradicionais } \\
\text { de atenção aos quadros } \\
\text { de depressão; o uso dos } \\
\text { dispositivos institucionais } \\
\text { e comunitários como parte } \\
\text { do projeto terapêutico } \\
\text { aponta para ações em saúde } \\
\text { mental que contemplam } \\
\text { a dimensão psicossocial. } \\
\text { A superação dos modelos } \\
\text { tradicionais de atendimento } \\
\text { à depressão e a construção } \\
\text { de formas de atenção } \\
\text { que utilizem recursos } \\
\text { psicossociais ainda estão } \\
\text { em curso, evidenciando que } \\
\text { a atenção integral precisa } \\
\text { ser mais bem consolidada } \\
\text { na Atenção Básica. }\end{array}$ & 2017 \\
\hline
\end{tabular}

Fonte: Autoria própria

\section{Discussão}

O processo de consolidação das práticas em saúde mental se confunde com o processo da efetivação do direito a integralidade da saúde. A necessidade de olhar para história, observando lutas, derrotas e conquistas que ocorreram, a fim de garantir a dignidade da população em receber do Estado o direito à saúde. Faz perceber a importância de olharmos para prática atual e refletir criticamente a produção do cuidado que, atenda de forma equitativa e universal o direito à saúde ${ }^{1}$.

A inclusão das ações de saúde mental na atenção básica é consequência de sucessivas conquistas de dois importantes movimentos:
Reforma Sanitária e Reforma Psiquiátrica brasileira. O primeiro garantiu o direito à saúde e o acesso universal e integral aos serviços e ações do setor. O segundo a reinserção social das pessoas em sofrimento psíquico que, desde muito tempo, foram excluídas da sociedade e do padrão de "normalidade" estabelecido ${ }^{9-14}$.

Como todo movimento social que se caracteriza pela incessante construção e reconstrução de possibilidades, a mais recente aposta do campo da saúde mental é incluirse no nível primário da atenção. Entretanto, as experiências relatadas não se direcionam ao sofrimento subjetivo relacionado a outras circunstâncias, como o próprio adoecer, ou decorrente da situação socioeconômica, agravada 
pela desigualdade, ou às situações corriqueiras do cotidiano, somente ao "transtorno psiquiátrico instalado"9.

As intervenções eficazes da assistência ao paciente com sofrimento psíquico, e as medidas a serem implementadas são relacionadas, principalmente, a própria (re)organização dos processos de trabalhos na APS, uma vez que, caracteriza-se por um conjunto de ações de saúde, no âmbito individual e coletivo, que abrange a promoção e a proteção da saúde, a prevenção de agravos, o diagnóstico, o tratamento, a reabilitação, a redução de danos e a manutenção da saúde, com o objetivo de desenvolver uma atenção integral que impacte na situação de saúde e autonomia das pessoas e nos determinantes e condicionantes de saúde das coletividades ${ }^{15}$.

A Estratégia Saúde da Família (ESF) visa à reorganização da atenção básica no país, de acordo com os preceitos do SUS. É tida pelo Ministério da Saúde do Brasil e gestores estaduais e municipais, representados respectivamente pelo Conselho Nacional de Secretários de Saúde (CONASS) e Conselho Nacional de Secretarias Municipais de Saúde (CONASEMS), como estratégia de expansão, qualificação e consolidação da atenção básica, por favorecer uma reorientação do processo de trabalho com maior potencial de aprofundar os princípios, diretrizes e fundamentos da atenção básica, de ampliar a resolutividade e impacto na situação de saúde das pessoas e coletividades, além de propiciar uma relação custo efetividade ${ }^{15}$.

Em todos os níveis da assistência, o acolhimento é a dimensão primária, do porteiro ao motorista, do auxiliar administrativo ao funcionário da limpeza, da equipe técnica, enfim, de todos que participam do processo de trabalho em serviço de saúde. Desta forma, acolher é indispensável passo para atendimento correto e bem sucedido ${ }^{16-18}$.

As intervenções em saúde mental devem promover possibilidades de modificar e qualificar modos de vida, orientando-se pela produção de vida e saúde, não se restringindo à cura de doenças. Isso significa acreditar que a vida pode ter várias formas de ser percebida, experimentada e vivida. Para tanto, é necessário olhar o sujeito em suas múltiplas dimensões, com seus desejos, anseios, valores e escolhas. Na APS, o desenvolvimento de intervenções em saúde mental é construído no cotidiano dos encontros entre profissionais e usuários, em que ambos criam novas ferramentas e estratégias para compartilhar e construir juntos o cuidado em saúde ${ }^{1}$.

Sendo assim, a eficácia das intervenções em saúde mentalé potencializada pelo acolhimento que os usuários recebem nas unidades de saúde, o que torna-se ferramenta para construção de vínculo entre usuário-profissional. $\mathrm{O}$ acolhimento não é simplesmente uma questão de escala em que se revezam profissionais, nem uma maneira mais racional de preencher as agendas. Portanto, o acolhimento é a aplicação cotidiana de um princípio fundamental, seja ao pedir informação mais corriqueira, seja ao trazer a mais fantasiosa expectativa, o usuário, quando aponta o problema, é um cidadão que exerce o direito de dirigir-se a um trabalhador de um serviço público ${ }^{16,19-23}$.

$\mathrm{O}$ acolhimento realizado nas unidades de saúde é um dispositivo para formação de vínculo e a prática de cuidado entre o profissional e o usuário. Por meio do acolhimento, a Equipe da Unidade de Saúde pode oferecer um espaço de escuta a usuários e a famílias, de modo que se sintam seguros e tranquilos para expressar suas aflições, dúvidas e angústias. A Unidade Básica de Saúde (UBS) está disponível para acolher, acompanhar, exigir e cuidar de forma compartilhada com outros serviços ${ }^{1}$.

A partir da Rede de Atenção Psicossocial (RAPS), em 2011, o Ministério da Saúde do Brasil propõe uma estruturação do modelo de cuidados em saúde a partir de ações da atenção básica, em um desenho ampliado, convocando a participação de diferentes equipes para trabalho articulado entre Atenção Básica em Saúde (Unidades Básicas de Saúde e equipes Consultório na Rua), atenção psicossocial especializada, atenção de urgência e emergência, atenção residencial de caráter transitório, atenção hospitalar, estratégias de desinstitucionalização e reabilitação psicossocial $^{17,24}$.

No Brasil, a atual Política de Saúde Mental, alinhada aos pressupostos da reforma psiquiátrica, propõe a progressiva substituição dos hospitais psiquiátricos por uma rede de serviços de atenção em saúde mental, constituída por Centros de Atenção Psicossocial (CAPS), ambulatórios de 
saúde mental, residências terapêuticas, centros de convivência e cultura, serviços de saúde mental, emergências psiquiátricas ${ }^{25-27}$.

Nesse sentido, surgiu a estratégia institucional conhecida por Apoio Matricial (AM), que assegura retaguarda especializada a equipes e profissionais encarregados da atenção em saúde mental. Além disso, favorece a corresponsabilização entre as equipes e a diversidade de ofertas terapêuticas através de profissional de saúde mental que acompanhe sistematicamente as Unidades Básicas de Saúde e permite conhecimento da demanda em saúde mental que chega à APS, bem como, aos usuários, as famílias e ao território, propondo que os casos sejam de responsabilidade mútua ${ }^{25,28-31}$.

Matriciamento ou apoio matricial é um novo modo de produzir saúde em que duas ou mais equipes, em um processo de construção compartilhada criam uma proposta de intervenção pedagógico-terapêutica. Tradicionalmente, os sistemas de saúde se organizam de forma vertical (hierárquica), com diferença de autoridade entre quem encaminha um caso e quem recebe, havendo transferência de responsabilidade ao encaminhar ${ }^{32}$.

A comunicação entre os dois ou mais níveis hierárquicos ocorre, muitas vezes, de forma precária e irregular, geralmente, por meio de informes escritos, como pedidos de parecer e formulários de contra referência que não oferecem resolubilidade. A nova proposta integradora visa transformar a lógica tradicional dos sistemas de saúde, como no caso de encaminhamentos, referências e contra referências, protocolos e centros de regulação. Os efeitos burocráticos pouco dinâmicos dessa lógica tradicional podem vir a ser atenuados por ações horizontais que integrem componentes e seus saberes nos diferentes níveis assistenciais $^{32}$.

\section{Conclusão}

Este estudo revelou que a literatura encontrada apresenta conceitos que convergem entre si, construindo categorias que possam servir de unidade de sentido para a reflexão do tema proposto, sendo base para discorrer e analisar as propostas que possam otimizar o cuidado do paciente em sofrimento mental.

Constatou-se que o acolhimento e o matriciamento foram os principais conceitos apresentados, sendo fundamentais para reorientação dos profissionais em relação ao cuidado de pacientes que apresentem algum tipo de sofrimento mental, seja ele ocasionado por um transtorno psicopatológico severo e/ou persistente, ou também por quadros adaptativos, onde o paciente não apresenta transtorno mental, porém, apresenta sofrimento psíquico decorrente de situações cotidianas (desemprego, violência e relacionamentos interpessoais).

O acolhimento como uma postura ética em relação ao usuário e seu sofrimento, serve como espaço para fornecer escuta qualificada e ampliada, indo ao encontro do conceito de integralidade, cuidado não fragmentando e o discurso do paciente, fortalecendo vínculo entre usuário e serviço de saúde. Pois, os pacientes acometidos por algum transtorno mental severo (esquizofrenia, por exemplo) acabam sendo estigmatizado pelos profissionais da atenção básica, sendo rotulados como àqueles pacientes que utilizam a unidade de saúde para "renovar receitas", deixando as outras necessidades deste paciente de lado.

O cuidado a esses pacientes acabam sendo reduzida a lógica do encaminhamento, e ao modelo biomédico, centralizando o cuidado a figura do médico. O papel do apoio matricial objetiva superar esta lógica reducionista, almejando que o cuidado possa ocorrer de uma forma resolutiva, e de responsabilidade do profissional da atenção básica, pois, diante das referências e contra referências, ocorre uma certa "terceirização" do cuidado, onde após o encaminhamento para serviço especializado, o profissional não sente que é mais responsável pelo paciente.

Assim, o cuidado ao paciente em sofrimento mental não necessita de inúmeros recursos tecnológicos/sofisticadas ou mudanças nos processos de trabalhos, pois, o foco do cuidado está na postura do profissional, independente da sua formação, e da organização da rede de atenção psicossocial como espaço de transição para que o paciente receba o cuidado que necessitar. 
Se faz necessário que os profissionais incorporem nas suas atividades cotidianas novos sentidos e novos olhares sobre esta população, apresentando a necessidade de Educação Permanente em Saúde aos profissionais e o Controle Social, no intuito de fortalecer os processos de trabalho na APS, como espaço democrático e fiscalizador da efetivação do direito à saúde.

\section{Referências}

1. Ministério da Saúde (BR). Secretaria de Atenção à Saúde. Departamento de Atenção Básica. Saúde mental. Departamento de Ações Programáticas Estratégicas. Brasília: Ministério da Saúde; 2013.

2. Nunes M, Juca VJ, Valentim CPB. Ações de saúde mental no Programa Saúde da Família: confluências e dissonâncias das práticas com os princípios das reformas psiquiátrica e sanitária. Cad Saúde Pública. 2007;23(10):2375-384

3. Pratta EMM, Santos MA. O processo saúde-doença e a dependência química: interfaces e evoluções. Psic Teor e Pesq. 2009;25(2):203-11.

4. Anjos MA, Carvalho PAL, Sena ELS, Ribeiro RMC. Acolhimento da pessoa em sofrimento mental na atenção básica para além do encaminhamento. Cad Brasileiros de Saúde Mental. 2015;7(16):27-40.

5. Cardoso L, Galera SAF. Mental health care today. Rev Esc Enferm USP. 2011;45(3):685-9.

6. Nery Filho A, Lorenzo C, Diz F. Usos e usuários de substancias psicoativas: considerações bioéticas. In: Brasil, Secretária Nacional de Políticas sobre Drogas, Prevenção dos problemas relacionados ao uso de drogas: capacitação para conselheiros e lideranças comunitárias/ Ministério da Justiça, Secretária Nacional de Políticas sobre Drogas, 6 ed. SENAD-MJ/NUTE-UFSC, Brasília: Ministério da Saúde; 2014.

7. Souza MT, Silva MD, Carvalho R. Revisão integrativa: o que é e como fazer. Einstein. 2010;8(1 Pt 1):102-06.

8. Goiás. Secretaria da Saúde (SES-GO). Superintendência de Educação em Saúde e Trabalho para o SUS (SESTSUS). Escola Estadual de Saúde Pública "Cândido Santiago" (ESAP-GO-GO). Metodologia da Pesquisa e do Trabalho Científico. Aurélio de Melo Barbosa - Goiânia: ESAP-GO; 2017.

9. Baroni DPM, Fontana LM. Ações em saúde mental na atenção primária no município de Florianópolis, Santa Catarina. Mental. 2009;7(12):15-37.
10. Souza LGS, Menandro MCS, Couto LLM, Schimith PB, Lima RP. Saúde mental na estratégia saúde da família: revisão da literatura brasileira. Saude soc. 2012;21(4):1022-034.

11. Moura RFS, Silva CRC. Saúde Mental na Atenção Básica: Sentidos Atribuídos pelos Agentes Comunitários de Saúde. Psicol cienc prof. 2015;35(1):199-10.

12. Bezerra E, Dimenstein M. Os CAPS e o trabalho em rede: tecendo o apoio matricial na atenção básica. Psicol cienc prof. 2008;28(3):632-45.

13. Prata NISS, Groisman D, Martins DA, Rabello ET, Mota FS, Jorge MA, et al. Saúde mental e atenção básica: território, violência e o desafio das abordagens psicossociais. Trab educ saúde. 2017;15(1):33-53.

14. Scardoelli MGC, Waidman MAP. "Grupo" de artesanato: espaço favorável à promoção da saúde mental. Esc Anna Nery. 2011;15(2):291-99.

15. Ministério da Saúde (BR). Secretaria de Atenção à Saúde. Departamento de Atenção Básica. Política Nacional de Atenção Básica. Brasília: Ministério da Saúde; 2012.

16. Minas Gerais. Secretaria de Estado de Saúde. Atenção em Saúde Mental. Marta Elizabeth de Souza. Belo Horizonte; 2006.

17. Moreira MIB, Onocko-Campos RT. Ações de saúde mental na rede de atenção psicossocial pela perspectiva dos usuários. Saude soc. 2017;26(2):462-74.

18. Rodrigues ES, Moreira MIB. A interlocução da saúde mental com atenção básica no município de Vitoria/ES. Saude Soc. 2012;21(3):599-11.

19. Lemke RA, Silva RAN. Itinerários de construção de uma lógica territorial do cuidado. Psicologia \& Sociedade. 2013;25(n.spe. 2):9-20.

20. Saffer DA, Barone LR. Em busca do comum: o cuidado do agente comunitário de saúde em Saúde Mental. Physis. 2017;27(3):813-33.

21. Lemke RA, Silva RAN. Um estudo sobre a itinerância como estratégia de cuidado no contexto das políticas públicas de saúde no Brasil. Physis. 2011;21(3):979-004.

22. Vecchia MD, Martins STF. Desinstitucionalização dos cuidados a pessoas com transtornos mentais na atenção básica: aportes para a implementação de ações. Interface Comunic, Saúde, Educ. 2009;13(28):151-64.

23. Costa FRM, Lima VV, Silva RF, Fioroni LN. Desafios do apoio matricial como prática educacional: a saúde mental na atenção básica. Interface. 2015;19(54):491-02.

24. Ministério da Saúde (BR). Portaria no 3.088, de 23 de dezembro de 2011. Institui a Rede de Atenção Psicossocial para pessoas com sofrimento ou transtorno mental e com necessidades decorrentes do uso de crack, álcool e outras drogas, no âmbito do Sistema Único de saúde (SUS). Diário Oficial da União, Brasília: Ministério da Saúde; 2011. 
25. Bezerra E, Dimenstein M. Os Caps e o trabalho em rede: tecendo o apoio matricial na atenção básica. Psicol Cienc Prof. 2008;28(3):632-45.

26. Motta CCL, More CLOO, Nunes CHSS. O atendimento psicológico ao paciente com diagnóstico de depressão na Atenção Básica. Ciênc saúde coletiva. 2017;22(3):911-20.

27. Delfini PSS, Sato MT, Antoneli PP, Guimarães POS. Parceria entre CAPS e PSF: o desafio da construção de um novo saber. Ciênc saúde coletiva. 2009;14(Suppl 1):1483-492.

28. Vecchia MD, Martins STF. Concepções dos cuidados em saúde mental por uma equipe de saúde da família, em perspectiva histórico-cultural. Ciênc saúde coletiva. 2009;14(1):183-93.

29. Abreu S, Miranda AAV, Murta SG. Programas Preventivos Brasileiros: Quem Faz e como É Feita a Prevenção em Saúde Mental? Psico-USF. 2016;21(1):163-77.

30. Paiva PC, Torrenté MON, Landim FLP, Branco JGO, Tamboril BCR, Cabral ALT. Sofrimento psíquico e a abordagem da comunidade na voz do agente comunitário de saúde. Rev Esc Enferm USP. 2016;50(n. spe):139-44.

31. Baroni DPM; Fontana LM. Ações em saúde mental na atenção primária no município de Florianópolis, Santa Catarina. Mental. 2009;7(12):15-37.

32. Guia prático de matriciamento em saúde mental. Ministério da Saúde: Centro de Estudo e Pesquisa em Saúde Coletiva. Brasília: Ministério da Saúde; 2011.

\section{Como citar este artigo:}

Gonçalves Junior M, Tobias GC, Teixeira CC. Saúde mental na atenção primária à saúde. Rev. Aten. Saúde. 2019;17(60): 101116. 